\title{
SEMINORMAL AND SUBNORMAL SUBGROUP LATTICES FOR TRANSITIVE PERMUTATION GROUPS
}

\author{
CHERYL E. PRAEGER
}

\author{
Dedicated to the memory of B. H. Neumann
}

(Received 5 May 2003; revised 17 November 2004)

Communicated by E. A. O’Brien

\begin{abstract}
Various lattices of subgroups of a finite transitive permutation group $G$ can be used to define a set of 'basic' permutation groups associated with $G$ that are analogues of composition factors for abstract finite groups. In particular $G$ can be embedded in an iterated wreath product of a chain of its associated basic permutation groups. The basic permutation groups corresponding to the lattice $\mathscr{L}$ of all subgroups of $G$ containing a given point stabiliser are a set of primitive permutation groups. We introduce two new subgroup lattices contained in $\mathscr{L}$, called the seminormal subgroup lattice and the subnormal subgroup lattice. For these lattices the basic permutation groups are quasiprimitive and innately transitive groups, respectively.
\end{abstract}

2000 Mathematics subject classification: primary 20B05, 20B10, $20 \mathrm{~B} 25$.

Keywords and phrases: transitive permutation group, primitive, quasiprimitive, innately transitive, subgroup lattice, subnormal subgroup.

\section{Introduction}

Primitive permutation groups have long been regarded as the 'building blocks' for finite transitive permutation groups since, for a given transitive permutation group $G$ on a finite set $\Omega$, there is a sequence $G_{1}, \ldots, G_{l}$ of primitive permutation groups such that $G$ can be embedded as a subgroup of the iterated wreath product $G_{1}, G_{2}, \cdots, G_{l}$ acting naturally on $\Omega$. Because of this embedding many questions about finite transitive permutation groups can be reduced to similar questions about primitive groups. Thus

This paper forms part of an Australian Research Council large grant project.

(C) 2006 Australian Mathematical Society 1446-7887/06 $\$$ A2.00+0.00 
the role of primitive groups for studying finite permutation groups is analogous to that of finite simple groups for the study of abstract finite groups. For some combinatorial applications, see for example [8], a larger family than the family of primitive groups is needed. In particular quasiprimitive and innately transitive permutation groups have arisen in this context. A primary aim of this paper is to demonstrate that the traditional analysis of transitive group actions, described below, can be replaced by a similar analysis that associates a set of quasiprimitive or innately transitive permutation groups with a given finite transitive group action. Most of the analysis applies to general (not necessarily finite) transitive group actions. The main results are Theorems 1.12 and 1.13 .

Transitive group actions For every group $G$ and proper subgroup $G_{0}$, the group $G$ acting by right multiplication induces a transitive permutation group on the set $\left[G: G_{0}\right]=\left\{G_{0} g \mid g \in G\right\}$ of right cosets of $G_{0}$ in $G$. Moreover, every transitive action of $G$ on a set of size at least 2 is equivalent to such a coset action, for some proper subgroup $G_{0}$. To emphasise both of the groups $G$ and $G_{0}$ in our notation, we will denote by $\operatorname{Trans}\left(G, G_{0}\right)$ the transitive permutation group induced by $G$ on $\left[G: G_{0}\right]$. In this action $G_{0}$ is the stabiliser of the 'point' $G_{0} \in\left[G: G_{0}\right]$, and $\operatorname{Trans}\left(G, G_{0}\right) \cong G /$ Core $_{G}\left(G_{0}\right)$ where Core ${ }_{G}\left(G_{0}\right)=\cap_{g \in G} G_{0}^{g}$, called the core of $G_{0}$ in $G$.

A fundamental problem that arises when studying any transitive action is that of determining the point partitions left invariant by the group (that is to say, partitions such that each element of the group permutes the blocks of the partition 'blockwise'). Such a partition is said to be non-trivial if it contains at least two blocks and each of its blocks contains at least two points. For the permutation group $\operatorname{Trans}\left(G, G_{0}\right)$, a nontrivial invariant partition $\mathscr{P}$ corresponds to a subgroup $H$ satisfying $G_{0}<H<G$. The blocks of $\mathscr{P}$ are indexed by the right cosets of $H$, namely the block of $\mathscr{P}$ corresponding to $H x$ is the set of right $G_{0}$-cosets contained in $H x$. Further, $\mathscr{P}$ gives rise to two transitive permutation groups, $\operatorname{Trans}(G, H)$ and $\operatorname{Trans}\left(H, G_{0}\right)$. The first of these $\operatorname{Trans}(G, H)$ is equivalent to the group induced by $G$ on the blocks of $\mathscr{P}$, while $\operatorname{Trans}\left(H, G_{0}\right)$ is equivalent to the group induced on one of the blocks. Moreover, $\operatorname{Trans}\left(G, G_{0}\right)$ can be embedded in the wreath product $\operatorname{Trans}\left(H, G_{0}\right) \geq \operatorname{Trans}(G, H)$ in its imprimitive action, see Theorem 1.6. We give a simple example of this situation below.

EXAMPLE 1.1. Let $\Omega=\{1,2, \ldots, 8\}, g=(12345678) \in \operatorname{Sym}(\Omega), G=\langle g\rangle \cong Z_{8}$, and $G_{0}=1$. Here $\operatorname{Trans}\left(G, G_{0}\right)$ is equivalent to the natural action of $G$ on $\Omega$ : for $i=0,1, \ldots, 7$, the coset $G_{0} g^{i}$ corresponds to the point $i+1$. For the subgroup $H=\left\langle g^{4}\right\rangle$, the $G$-invariant partition of $\left[G: G_{0}\right]$, as described above, corresponds to the partition $\mathscr{P}=\{B, C, D, E\}=\{1,5|2,6| 3,7 \mid 4,8\}$ of $\Omega$, and the 
groups $\operatorname{Trans}(G, H)$ and $\operatorname{Trans}\left(H, G_{0}\right)$ are equivalent to $\langle(B, C, D, E)\rangle \cong Z_{4}$ and $\langle(1,5)\rangle \cong Z_{2}$ respectively, so that $\operatorname{Trans}\left(G, G_{0}\right) \leq Z_{2}<Z_{4}<\operatorname{Sym}\left(\left[G: G_{0}\right]\right)$.

If there is no non-trivial partition of $\left[G: G_{0}\right]$ preserved by $\operatorname{Trans}\left(G, G_{0}\right)$, or equivalently, if the stabiliser $G_{0}$ is a maximal subgroup of $G$, then $\operatorname{Trans}\left(G, G_{0}\right)$ is said to be primitive; and otherwise $\operatorname{Trans}\left(G, G_{0}\right)$ is imprimitive. Suppose that $G_{0}<H<G$. If there is a non-trivial invariant point partition for one of $\operatorname{Trans}(G, H)$ or Trans $\left(H, G_{0}\right)$, or equivalently if either $G_{0}$ is not maximal in $H$, or $H$ is not maximal in $G$, then the same analysis leads to a chain of subgroups of length three from $G_{0}$ to $G$, and an embedding of $\operatorname{Trans}\left(G, G_{0}\right)$ into an iterated wreath product with three 'factors'. This happens with the groups in Example 1.1.

EXAMPLE 1.2. For the group $G$ in Example 1.1, $\{X, Y\}=\{B, D \mid C, E\}$ is a $\operatorname{Trans}(G, H)$-invariant partition of $\mathscr{P}$, corresponding to the subgroup $K=\left\langle g^{2}\right\rangle$ satisfying $H<K<G$. The groups $\operatorname{Trans}(G, K)$ and $\operatorname{Trans}(K, H)$ are equivalent to $\langle(X, Y)\rangle$ and $\langle(B, D)\rangle$ respectively. Thus we have $G_{0}<H<K<G$ and $\operatorname{Trans}\left(G, G_{0}\right) \leq Z_{2} \gtrless Z_{2}: Z_{2} \leq \operatorname{Sym}\left(\left[G: G_{0}\right]\right)$.

For arbitrary transitive group actions, on iterating this process we find, for each subgroup chain of length $k$ from $G_{0}$ to $G$, an embedding of $\operatorname{Trans}\left(G, G_{0}\right)$ into an iterated wreath product with $k$ transitive permutation groups as 'factors'. If each subgroup of the chain is maximal in the next largest member of the chain, then the corresponding transitive groups are primitive, and the chain can be refined no further. Moreover, in this case we have an embedding of $\operatorname{Trans}\left(G, G_{0}\right)$ into an iterated wreath product of primitive groups. This is the situation in Example 1.2 where we have three primitive groups, each equivalent to the primitive group of order two acting on two points.

In the case where $\left|G: G_{0}\right|$ is finite we can always find a maximal subgroup chain from $G_{0}$ to $G$, and hence an embedding of $\operatorname{Trans}\left(G, G_{0}\right)$ into a wreath product of primitive groups. However, for a complete understanding of the primitive groups involved in $\operatorname{Trans}\left(G, G_{0}\right)$ we need in general to examine more than a single maximal chain of subgroups from $G_{0}$ to $G$. The reason is that different primitive groups may occur for different maximal subgroup chains, as illustrated in the next example.

EXAMPLE 1.3. Let $X=\{1,2, \ldots, n\}, G=\operatorname{Sym}(X)$ and $G_{0}=\operatorname{Sym}(\{3, \ldots, n\})$, so that $\operatorname{Trans}\left(G, G_{0}\right)$ is equivalent to the action of $G$ on $\Omega=\{(i, j) \mid i, j \in X, i \neq j\}$. There are precisely three maximal subgroup chains from $G_{0}$ to $G$, namely three chains of the form $G_{0}<H<G$ giving rise to embeddings of $\operatorname{Trans}\left(G, G_{0}\right) \cong G$ into $W_{H}:=\operatorname{Trans}\left(H, G_{0}\right) \geq \operatorname{Trans}(G, H)$. The subgroup $H$ is one of the point stabilisers $G_{1}$ or $G_{2}$ in the natural action of $G$ on $X$, or is the setwise stabiliser $G_{(1.2)}$. If $H=G_{1}$ or $G_{2}$, then $W_{H}=S_{n-1}, S_{n}$ with $S_{n-1}$ and $S_{n}$ in their natural actions on $n-1$ and $n$ 
points respectively. On the other hand, if $H=G_{\{1,2\}}$ then $W_{H}=S_{2}$ ? $S_{n}$, this time with $S_{n}$ acting primitively on the $n(n-1) / 2$ unordered pairs from $X$.

To obtain a full understanding of the primitive permutation groups associated with a given finite transitive group $\operatorname{Trans}\left(G, G_{0}\right)$ we need to study the lattice of subgroups of $G$ containi.g $G_{0}$. This is the traditional framework for studying transitive group actions, and has proved effective in many applications for reducing a problem concerning an algebraic or combinatorial structure admitting a transitive automorphism group into a similar problem for such a structure admitting a primitive group, see for example $[4,10]$.

However, in several applications in graph theory and geometry (see, for example, $[5,9])$ there is no reduction possible to the primitive case, but instead it is possible to reduce some problems to cases where the relevant permutation group is quasiprimitive (see Definition 1.11). By a similar analysis to that above we will associate a family of quasiprimitive groups with any given finite transitive group action.

Subgroup lattices and basic permutation groups A subgroup lattice for a group $G$ is a non-empty set of subgroups of $G$, partially ordered by inclusion, such that every pair of groups in the lattice has a least upper bound (also called a supremum) and a greatest lower bound (also called an infimum) in the lattice. We will study various kinds of subgroup lattices, and with each type we will associate a family of permutation groups that we call basic. In particular, we will prove that each finite transitive permutation group can be embedded in an iterated wreath product of basic permutation groups. We will discuss three types of lattices. The first is the lattice of all subgroups containing a given subgroup; its basic groups are the primitive groups. The other two are the seminormal and subnormal lattices, and for these the basic permutation groups are the quasiprimitive, and innately transitive permutation groups, respectively (see Definition 1.11 ).

A non-empty subset $\mathscr{L}_{1}$ of a subgroup lattice $\mathscr{L}_{2}$ is a sublattice of $\mathscr{L}_{2}$ if, for all $H, K \in \mathscr{L}_{1}$, the least upper bound and greatest lower bound of $H$ and $K$ in $\mathscr{L}_{2}$ both lie in $\mathscr{L}_{1}$. For some of the subgroup lattices $\mathscr{L}_{1}, \mathscr{L}_{2}$ studied in this paper, $\mathscr{L}_{1}$ may be a subset, but not a sublattice, of $\mathscr{L}_{2}$.

DEFINITION 1.4. Let $\mathscr{X}$ be a family of pairs $\left(G, G_{0}\right)$, where $G$ is a group and $G_{0}$ is a proper subgroup of $G$. A subgroup lattice function $\mathscr{L}$ on $\mathscr{X}$ is a function on $\mathscr{X}$ such that, for $\left(G, G_{0}\right) \in \mathscr{X}$, the output $\mathscr{L}\left(G, G_{0}\right)$ is a subgroup lattice that contains $G$ and is a subset of the lattice of all subgroups of $G$ containing $G_{0}$. Such a function $\mathscr{L}$ is called strong if, whenever $H, K \in \mathscr{L}\left(G, G_{0}\right)$ with $H<K$, then $(K, H) \in \mathscr{X}$ and $\mathscr{L}(K, H)=\left\{M \in \mathscr{L}\left(G, G_{0}\right) \mid H \leq M \leq K\right\}$.

If $\mathscr{L}$ is a subgroup lattice function, and $H, K \in \mathscr{L}\left(G, G_{0}\right)$, then we say that $K$ 
covers $H$ in $\mathscr{L}\left(G, G_{0}\right)$ if $H<K$ and there is no subgroup $L$ such that $H<L<K$ and $L \in \mathscr{L}\left(G, G_{0}\right)$. If $\mathscr{L}$ is a strong subgroup lattice function then $K$ covers $H$ in $\mathscr{L}\left(G, G_{0}\right)$ if and only if $\mathscr{L}(K, H)=\{K, H\}$. Components and basic groups for $\mathscr{L}$ are defined as follows.

DEFINITION 1.5. Let $\mathscr{L}$ be a subgroup lattice function on $\mathscr{X}$, and let $\left(G, G_{0}\right) \in \mathscr{X}$. If $H, K \in \mathscr{L}\left(G, G_{0}\right)$ and $H<K$ then $\operatorname{Trans}(K, H)$ is called an $\mathscr{L}$-component of $\operatorname{Trans}\left(G, G_{0}\right)$. If in addition $K$ covers $H$ in $\mathscr{L}\left(G, G_{0}\right)$, then $\operatorname{Trans}(K, H)$ is an $\mathscr{L}$-basic component of $\operatorname{Trans}\left(G, G_{0}\right)$.

The following result about embedding a transitive group in an iterated wreath product of its components follows immediately from the embedding theorem for imprimitive permutation groups, see for example [3, Theorem 8.5]. A subgroup chain $G_{0}<\cdots<G_{l}=G$ is $\mathscr{L}$-maximal if each of the $G_{i}$ lies in $\mathscr{L}\left(G, G_{0}\right)$, and each $G_{i}$ covers $G_{i-1}$ in $\mathscr{L}\left(G, G_{0}\right)$.

THEOREM 1.6. Let $\mathscr{L}$ be a subgroup lattice function on some set $\mathscr{X}$, and suppose that $\left(G, G_{0}\right) \in \mathscr{X}$ and $G_{0}<\cdots<G_{l}=G$ is a finite chain of subgroups in $\mathscr{L}\left(G, G_{0}\right)$, where $l \geq 1$. Then, $\Omega:=\left[G: G_{0}\right]$ can be identified with $\prod_{i=1}^{l}\left[G_{i}: G_{i-1}\right]$ in such a way that

$$
\operatorname{Trans}\left(G, G_{0}\right) \leq \operatorname{Trans}\left(G_{1}, G_{0}\right) \imath \cdots \imath \operatorname{Trans}\left(G_{l}, G_{l-1}\right) \leq \operatorname{Sym}(\Omega) .
$$

Moreover, if the chain is $\mathscr{L}$-maximal then, for each $i=1, \ldots, l$, Trans $\left(G_{i}, G_{i-1}\right)$ is an $\mathscr{L}$-basic component of $\operatorname{Trans}\left(G, G_{0}\right)$.

Primitive groups arise naturally as basic permutation groups corresponding to the most natural subgroup lattice function Sub, where $\operatorname{Sub}\left(G, G_{0}\right)=\left\{H \mid G_{0} \leq H \leq\right.$ $G\}$ is the lattice of all subgroups of $G$ containing $G_{0}$. Moreover, Sub is defined on the family $\mathscr{X}_{\text {all }}$ of all pairs $\left(G, G_{0}\right)$ where $G$ is a group and $G_{0}$ is a proper subgroup. The following theorem follows immediately from the standard theory of transitive permutation groups, see, for example, [6, Theorem 1.5A], and encapsulates the discussion in the previous subsection.

THEOREM 1.7. The function Sub is a strong subgroup lattice function on $\mathscr{X}_{\mathrm{all}}$, and if $G_{0}<G$ then every Sub-basic component of $\operatorname{Trans}\left(G, G_{0}\right)$ is primitive. Moreover, each primitive permutation group $\operatorname{Trans}(K, H)$ occurs, for example, as the unique Sub-basic component of $\operatorname{Trans}(K, H)$.

REMARK 1.8. Finally in this subsection we mention a direction in which the theory of this paper might be developed. In [1, Section 3] a generalised wreath product $\prod_{(\mathscr{I} . \leq)}\left(G_{i}, \Delta_{i}\right)$ of transitive permutation groups $G_{i}$ on $\Delta_{i}(i \in \mathscr{I})$ was constructed, 
based on a partially ordered set $(\mathscr{I}, \leq)$ satisfying the maximal condition (every nonempty subset of $\mathscr{I}$ has a maximal element). The generalised wreath product was defined as a permutation group on $\Delta:=\prod_{i \in \mathscr{I}} \Delta_{i}$, and was shown to be transitive and to leave invariant a 'poset block structure' on $\Delta$, see [1, Lemma 9 and Section 4]. The construction reduces to the iterated wreath product of Theorem 1.6 in the case where $(\mathscr{I}, \leq)$ is a chain. It would be interesting to know the extent to which the theory of this paper extends to generalised wreath products. When is there a partially ordered set $\mathscr{I}$ corresponding to a subgroup lattice $\mathscr{L}\left(G, G_{0}\right)$ ? When is there an embedding of $\operatorname{Trans}\left(G, G_{0}\right)$ into the corresponding generalised wreath product?

Seminormal and subnormal lattices Now we define the subnormal and seminormal lattice functions, and discuss their basic groups. A subgroup $N$ of a group $G$ is subnormal in $G$, denoted $N \triangleleft \triangleleft G$, if there is a finite chain $N_{0}=N<\cdots<N_{l}=G$ such that, for each $i<l, N_{i}$ is a normal subgroup of $N_{i+1}$, denoted $N_{i} \triangleleft N_{i+1}$; such a chain is called a subnormal chain for $N$. As in [11] we call $N$ a composition subgroup of $G$ if there is a subnormal chain from $N$ to $G$ in which all the factor groups $N_{i+1} / N_{i}$ are simple; and we call a subnormal chain with this additional property a composition series from $N$ to $G$. Clearly a composition subgroup is subnormal, but the converse does not hold in general (see [7]).

DEFINITION 1.9. (a) For $G_{0}<G$, define $\operatorname{Subnorm}\left(G, G_{0}\right)$ as the set of all subgroups of the form $G_{0} N$, where $N$ is a $G_{0}$-invariant composition subgroup of $G$.

(b) For $G_{0}<G$, and for $G_{0} \leq H \leq K \leq G$, a $G_{0}$-seminormal chain of length $l$ from $H$ to $K$ is a subgroup chain $H=H_{0} \leq H_{1} \leq \cdots \leq H_{l}=K$, such that $l \geq 0$ and, if $0 \leq i<l$ then $H_{i}=G_{0} N_{i}$ for some $N_{i} \triangleleft H_{i+1}$. The pair $(K, H)$ is called $G_{0}$-seminormal if there exists a $G_{0}$-seminormal chain from $H$ to $K$, and it is said to have length $l$, where $l \geq 0$, if $l$ is the minimum length of such a chain. Define Seminorm $\left(G, G_{0}\right)$ as the set of all subgroups $H$ containing $G_{0}$ such that $(G, H)$ is $G_{0}$-seminormal.

We prove in Lemma 2.1 that, for a $G_{0}$-seminormal chain as in part (b), we may take $N_{i}=$ Core $_{H_{i+1}}\left(H_{i}\right)$. Also,

$$
\operatorname{Subnorm}\left(G, G_{0}\right) \subseteq \operatorname{Seminorm}\left(G, G_{0}\right) \subseteq \operatorname{Sub}\left(G, G_{0}\right)
$$

(see Proposition 3.2), and we have $G \in \operatorname{Subnorm}\left(G, G_{0}\right)$ (since $G=G_{0} G$ ), $G_{0} \in \operatorname{Seminorm}\left(G, G_{0}\right)$ (since $G_{0}=G_{0}\left\{1_{G}\right\}$ ), and if $G_{0}$ contains a $G_{0}$-invariant composition subgroup $N$ of $G$ then also $G_{0}=G_{0} N \in \operatorname{Subnorm}\left(G, G_{0}\right)$. Equality can occur among the above displayed inclusions, for example, if $G_{0}$ is maximal in $G$ and $\left\{1_{G}\right\}$ is a composition subgroup, then $\operatorname{Sub}\left(G, G_{0}\right)=\operatorname{Seminorm}\left(G, G_{0}\right)=$ Subnorm $\left(G, G_{0}\right)=\left\{G, G_{0}\right\}$. However in general these sets of subgroups need not be equal, as we illustrate in the following examples. 
EXAMPLE 1.10. (a) Let $G=S_{3}$, and $G_{0}=\left\{1_{G}\right\}$. Then $\operatorname{Sub}\left(G, G_{0}\right)$ contains all the subgroups of $G$ and properly contains the set $\operatorname{Seminorm}\left(G, G_{0}\right)=$ Subnorm $\left(G, G_{0}\right)=\left\{\left\{1_{G}\right\}, A_{3}, G\right\}$.

(b) Let $G=A \times S<\operatorname{Sym}(X)$, where $X=\{1,2,3,4,5,6,7,8\}, A \cong A_{5}$ is the alternating group acting on $\{1,2,3,4,5\}$, and $S \cong S_{3}$ acting on $\{6,7,8\}$. Let

$$
G_{0}=\langle(12345),(25)(34)(67)\rangle \cong D_{10} .
$$

Since Core $_{G}\left(G_{0}\right)=\left\{1_{G}\right\}$, we have $\operatorname{Trans}\left(G, G_{0}\right) \cong G$ of degree $\left|G: G_{0}\right|=36$. There are six subnormal subgroups of $G$, namely $\left\{1_{G}\right\}, A, S, S^{\prime}, A \times S^{\prime}, G$. Each is normal in $G$, and hence is $G_{0}$-invariant. Thus, setting $Z=\langle(67)\rangle$ and $D=$ $\langle(12345),(25)(34)\rangle \cong D_{10}$,

$$
\operatorname{Subnorm}\left(G, G_{0}\right)=\left\{G_{0}, G_{0} A=A \times Z, G_{0} S^{\prime}, G_{0} S=D \times S, G\right\},
$$

while $\operatorname{Seminorm}\left(G, G_{0}\right)=\operatorname{Sub}\left(G, G_{0}\right)$, and consists of $\operatorname{Subnorm}\left(G, G_{0}\right)$ and one additional subgroup $G_{0} Z=G_{0} D=D \times Z$.

We shall prove that Seminorm and Subnorm are subgroup lattice functions on appropriate sets $\mathscr{X}$, and that the basic components for Seminorm and Subnorm are quasiprimitive and innately transitive respectively, where we now define these concepts.

DEFINITION 1.11. (a) A transitive permutation group $G$ is quasiprimitive if every non-identity normal subgroup of $G$ is transitive.

(b) A transitive permutation group $G$ is innately transitive if $G$ has at least one transitive minimal normal subgroup.

If a transitive permutation group $G$ on $\Omega$ is not quasiprimitive then it has a nonidentity intransitive normal subgroup $N$. In this case $G_{\omega}<G_{\omega} N<G$ for $\omega \in \Omega$, and hence $G$ is imprimitive. Thus every primitive permutation group is quasiprimitive, and it follows immediately from the definition that every finite quasiprimitive permutation group is innately transitive.

The main outcome of the paper is that all the above theory applies to Seminorm and Subnorm. Although these functions can be applied to all pairs in $\mathscr{X}_{\text {all }}$, we have been able to prove only that Seminorm and Subnorm are subgroup lattice functions on the families

$\mathscr{X}_{\text {finite }}^{\text {Seminorm }}$ and $\mathscr{X}_{\text {finite }}^{\text {Subnorm }}$ consisting of those pairs $\left(G, G_{0}\right)$ for which $\operatorname{Seminorm}\left(G, G_{0}\right)$ and $\operatorname{Subnorm}\left(G, G_{0}\right)$, respectively, are finite.

Clearly, $\mathscr{X}_{\text {finite }}^{\text {Seminom }} \subseteq \mathscr{X}_{\text {finite }}^{\text {Subnorm }} \subset \mathscr{X}_{\text {all }}$. It is possible, however, that the theory may extend to larger subsets of $\mathscr{X}_{\mathrm{all}}$. 
THEOREM 1.12. The function Seminorm is a subgroup lattice function on the set

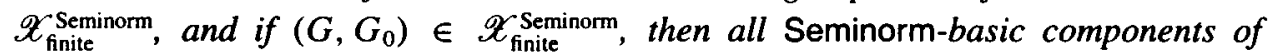
$\operatorname{Trans}\left(G, G_{0}\right)$ are quasiprimitive. Moreover if $\operatorname{Trans}(K, H)$ is an arbitrary quasiprimitive group, then $(K, H) \in \mathscr{X}_{\text {finite }}^{\text {Seminorm }}$ and $\operatorname{Seminorm}(K, H)=\{K, H\}$.

THEOREM 1.13. The function Subnorm is a subgroup lattice function on the set

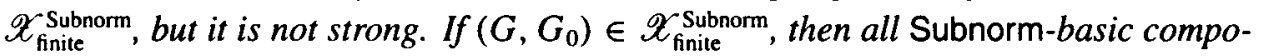
nents of $\operatorname{Trans}\left(G, G_{0}\right)$ are innately transitive. Moreover,

(a) Subnorm $\left(G, G_{0}\right)=\left\{G, G_{0}\right\}$ if and only if $\operatorname{Trans}\left(G, G_{0}\right)$ is quasiprimitive;

(b) each finite innately transitive group occurs as a Subnorm-basic component of $\operatorname{Trans}\left(G, G_{0}\right)$, for some $\left(G, G_{0}\right) \in \mathscr{X}_{\text {finite }}^{\text {Subnorm }}$.

Example 1.14 below demonstrates that $\operatorname{Subnorm}\left(G, G_{0}\right)$ is not always a sublattice of $\operatorname{Sub}\left(G, G_{0}\right)$, and that the function Subnorm is not strong.

EXAMPLE 1.14. Let $A, B$ be finite nonabelian simple groups, let $\left\{1_{A}\right\}<C<A$ and $\left\{1_{B}\right\}<D<B$ such that $C \cong D$, and let $\varphi: C \rightarrow D$ be an isomorphism. Define $G=A \times B$ and $G_{0}=\{(x, \varphi(x)) \mid x \in C\}$. Then each subnormal subgroup of $G$ is normal and the normal subgroups are $\left\{1_{G}\right\}, A, B, G$. Thus

$$
\operatorname{Subnorm}\left(G, G_{0}\right)=\left\{G_{0}, G_{0} A=A \times D, G_{0} B=C \times B, G\right\}
$$

The greatest lower bound for $G_{0} A$ and $G_{0} B$ in $\operatorname{Subnorm}\left(G, G_{0}\right)$ is therefore $G_{0}$, whereas $G_{0} A \cap G_{0} B=C \times D$ properly contains $G_{0}$. Thus Subnorm $\left(G, G_{0}\right)$ is not a sublattice of $\operatorname{Sub}\left(G, G_{0}\right)$.

Note also that the Subnorm-basic components $\operatorname{Trans}\left(G_{0} A, G_{0}\right) \cong A \times D$ and $\operatorname{Trans}\left(G_{0} B, G_{0}\right) \cong C \times B$ of $\operatorname{Trans}\left(G, G_{0}\right)$ are both innately transitive but not quasiprimitive. Moreover, Subnorm $\left(G_{0} A, G_{0}\right)$ contains $\left\{G_{0} A, C \times D, G_{0}\right\}$, whereas $G_{0} A$ covers $G_{0}$ in Subnorm $\left(G, G_{0}\right)$. Thus the function Subnorm is not strong.

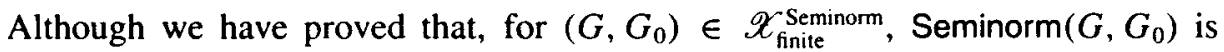
a subgroup lattice, we have been unable to prove that it is always a sublattice of $\operatorname{Sub}\left(G, G_{0}\right)$. However we have not found an example where it fails to be. More precisely, we prove in Section 2 that, for any $\left(G, G_{0}\right) \in \mathscr{X}_{\text {all }}$, the infimum of two subgroups $H, K$ in Seminorm $\left(G, G_{0}\right)$ is their intersection $H \cap K$, and if $\left(G, G_{0}\right) \in$ $\mathscr{X}_{\text {finite }}^{\text {Seminom }}$ we prove that $H, K$ have a supremum in $\operatorname{Seminorm}\left(G, G_{0}\right)$. However in the latter case we have been unable either to prove or to disprove that their supremum is always $\langle H, K\rangle$ (see Propositions 2.3 and 2.4).

Similarly (or perhaps, by contrast), for any $\left(G, G_{0}\right) \in \mathscr{X}_{\text {all }}$, we prove that the supremum of two subgroups $H, K$ in $\operatorname{Subnorm}\left(G, G_{0}\right)$ is $\langle H, K\rangle$ and, if $\left(G, G_{0}\right) \in$ $\mathscr{X}_{\text {finite }}^{\text {-Subn, }}$, then we prove in Proposition 3.2 that $H$ and $K$ have an infimum in 
TABLE 1. Summary: for $\mathscr{L}_{0}$ finite and $H, K \in \mathscr{L}_{0}$.

\begin{tabular}{|ccccc|}
\hline $\mathscr{L}_{0}$ & $H \cap K$ & $\langle H, K\rangle$ & $G_{0}$-basic & $\mathscr{L}_{0}=\left\{G, G_{0}\right\} \Longleftrightarrow$ \\
& $\in \mathscr{L}_{0} ?$ & $\in \mathscr{L}_{0} ?$ & components & Trans $\left(G, G_{0}\right)$ is \\
\hline $\operatorname{Sub}\left(G, G_{0}\right)$ & yes & yes & primitive & primitive \\
Seminorm $\left(G, G_{0}\right)$ & yes & $? ? ?$ & quasiprimitive & quasiprimitive \\
Subnorm $\left(G, G_{0}\right)$ & not & yes & innately & quasiprimitive \\
& always & & transitive & \\
\end{tabular}

Subnorm $\left(G, G_{0}\right)$. In this case we know from Example 1.14 that the infimum may sometimes be a proper subgroup of $H \cap K$.

We summarise in Table 1 the properties of the three subgroup lattice functions studied in this paper, and we record below the major unresolved issues.

ProBlem 1.15. Describe the pairs $\left(G, G_{0}\right) \in \mathscr{X}_{\text {all }}$ (if such exist) for which

(1) Seminorm $\left(G, G_{0}\right)$ or Subnorm $\left(G, G_{0}\right)$ is not a subgroup lattice; and

(2) Seminorm $\left(G, G_{0}\right)$ or Subnorm $\left(G, G_{0}\right)$ is a subgroup lattice, but not a sublattice of $\operatorname{Sub}\left(G, G_{0}\right)$.

PROBlem 1.16. Decide whether or not Seminorm is a strong subgroup lattice function on $\mathscr{X}_{\text {finite }}^{\text {Seminorm }}$.

PROBLEM 1.17. Find the largest subsets of $\mathscr{X}_{\text {all }}$ on which Seminorm and Subnorm are subgroup lattice functions.

The proof that, for $\left(G, G_{0}\right) \in \mathscr{X}_{\text {finite }}^{\text {Seminorm }}$, $\operatorname{Seminorm}\left(G, G_{0}\right)$ is a subgroup lattice is delicate but elementary, and this part of the proof is given in Section 2, where we investigate some of the properties of seminormal subgroup chains. It was proved in 1939 by Wielandt (see [11, Theorem 1.1.5]) that the partially ordered set of all composition subgroups of a group $G$ is a sublattice of the lattice of all subgroups of $G$. This is the major tool used in Section 3 to prove that, for any subgroup $G_{0}$ of a group $G, \operatorname{Subnorm}\left(G, G_{0}\right)$ is a subgroup lattice contained in $\operatorname{Seminorm}\left(G, G_{0}\right)$. We use the characterisation of finite innately transitive permutation groups in [2] to prove that each finite innately transitive group occurs as a Subnorm-basic component of some finite $\operatorname{Trans}\left(G, G_{0}\right)$.

\section{The seminormal lattice function}

Throughout the rest of the paper let $\left(G, G_{0}\right) \in \mathscr{X}_{\text {all }}$, that is, $G$ is a group with a proper subgroup $G_{0}$. It follows from Definition 1.9 that, for $G_{0} \leq H \leq K \leq G$, 
$(K, H)$ is $G_{0}$-seminormal of length at most 1 if and only if $H=G_{0} N$ for some normal subgroup $N$ of $K$. For convenience we will call such a pair $G_{0}$-normal.

LEMMA 2.1. If $(K, H)$ is $G_{0}$-normal, then there is a unique largest subgroup $N$ such that $H=G_{0} N$ and $N$ is normal in $K$, namely $N=\operatorname{Core}_{K}(H)=\bigcap_{x \in K} H^{x}$.

ProOF. We have that $H=G_{0} M$ for some normal subgroup $M$ of $K$. Set $N=$ Core $_{K}(H)$. Then $N$ is normal in $K$ and $N \leq H$, so $G_{0} N \leq H$. On the other hand, since $M$ is normal in $K, H^{x}$ contains $M^{x}=M$ for each $x \in K$, and hence $M \leq N$. Thus $H=G_{0} M \leq G_{0} N$ and so $H=G_{0} N$ and $N$ is the largest such subgroup normal in $K$.

The next simple property of $G_{0}$-normal pairs is important.

LEMMA 2.2. If $(K, H)$ is $G_{0}$-normal and $G_{0} \leq L \leq K$, then $(L, H \cap L)$ is also $G_{0}$-normal. If also $(K, L)$ is $G_{0}$-normal, then $H L$ is a subgroup and $(K, H L)$, $(H L, L),(H L, H)$, and $(H, H \cap L)$ are all $G_{0}$-normal.

Proof. By definition, $H=G_{0} N$, where $N$ is a normal subgroup of $K$. Thus $H \cap L=\left(G_{0} N\right) \cap L$ and we claim that $\left(G_{0} N\right) \cap L=G_{0}(N \cap L)$. Since $G_{0} \subseteq L$, we have $G_{0}(N \cap L) \subseteq\left(G_{0} N\right) \cap L$. Suppose now that $y=g x \in\left(G_{0} N\right) \cap L$, with $g \in G_{0}$ and $x \in N$. Then $x=g^{-1} y \in N \cap L$ (since $L$ contains $G_{0}$ ). Hence $y=g x \in G_{0}(N \cap L)$, proving the claim. Since $N \cap L$ is normal in $L$ it follows that $(L, H \cap L)$ is $G_{0}$-normal.

Now suppose that $(K, L)$ is also $G_{0}$-normal, so $L=G_{0} M$ for some normal subgroup $M$ of $K$. Then the argument of the previous paragraph applied to $(K, L)$ proves that $H \cap L=G_{0}(H \cap M)$, that $H \cap M$ is normal in $H$, and that $(H, H \cap L)$ is $G_{0}$-normal.

Now $H L=\left(G_{0} N\right)\left(G_{0} M\right)=G_{0} N M$, since $G_{0}$ normalises $N$. Since $N M$ is a normal subgroup of $K$, it follows that $H L$ is a subgroup of $K$ and $(K, H L)$ is $G_{0}$-normal. That $(H L, L)$ and $(H L, H)$ are $G_{0}$-normal follows by applying the first assertion to the subgroup $H L$ and $G_{0}$-normal pairs $(K, L)$ and $(K, H)$ respectively.

Recalling from Definition 1.9 the definition of $G_{0}$-seminormal chains and subgroup pairs, we observe that concatenations of $G_{0}$-seminormal chains are again $G_{0}$ seminormal. Thus if $G_{0}<L<H<K \leq G$, and if $(K, H)$ and $(H, L)$ are $G_{0}$-seminormal of lengths $h, l$ respectively, then $(K, L)$ is also $G_{0}$-seminormal of length at most $h+l$. Note that a $G_{0}$-seminormal pair $(K, H)$ has length 0 if and only if $K=H$. 
PROPOSITION 2.3. Let $\left(G, G_{0}\right) \in \mathscr{X}_{\text {all }}$, and let $G_{0} \leq H \leq K \leq G$ and $G_{0} \leq$ $L \leq K \leq G$ be such that $(K, H),(K, L)$ are $G_{0}$-seminormal of lengths $h$ and $l$ respectively. Let $H=H_{0} \leq H_{1} \leq \cdots \leq H_{h}=K$ be a $G_{0}$-seminormal chain.

(a) If $l=1$, then $(H \cap L) \leq\left(H_{1} \cap L\right) \leq \cdots \leq\left(H_{h} \cap L\right)=L$ is also a $G_{0}$-seminormal chain, so $(L, H \cap L)$ is $G_{0}$-seminormal of length at most $h$.

(b) For any $h, l,(H, H \cap L),(L, H \cap L)$ and $(K, H \cap L)$ are $G_{0}$-seminormal pairs of lengths at most $l, h$ and $h+l$ respectively.

(c) If $H, L \in \operatorname{Seminorm}\left(G, G_{0}\right)$, then $H \cap L \in \operatorname{Seminorm}\left(G, G_{0}\right)$.

ProOF. For $0 \leq i<h, H_{i}=G_{0} N_{i}$ for some normal subgroup $N_{i}$ of $H_{i+1}$. First we prove part (a). Suppose that $l=1$, so $L=G_{0} M$ for some normal subgroup $M$ of $K$. Applying Lemma 2.2 to the $G_{0}$-normal pairs $\left(K, H_{h-1}\right)$ and $(K, L)$, we find that $\left(H_{h-1}, H_{h-1} \cap L\right)$ and $\left(L, H_{h-1} \cap L\right)$ are both $G_{0}$-normal. Similarly, applying Lemma 2.2 to the $G_{0}$-normal pairs $\left(H_{h-1}, H_{h-2}\right)$ and $\left(H_{h-1}, H_{h-1} \cap L\right)$, we find that $\left(H_{h-2}, H_{h-2} \cap L\right)$ and $\left(H_{h-1} \cap L, H_{h-2} \cap L\right)$ are both $G_{0}$-normal. Continuing in this way we deduce that $(H \cap L) \leq\left(H_{1} \cap L\right) \leq \cdots \leq\left(H_{h} \cap L\right)=L$ is indeed a $G_{0}$-seminormal chain of length $h$, proving part (a).

If either $h$ or $l$ is zero, then the assertions of part (b) follow from the definitions of $h$ and $l$. Thus we may assume that $h$ and $l$ are both positive, so $h+l \geq 2$. We shall prove part (b) by induction on $h+l$. Let $L=L_{0} \leq L_{1} \leq \cdots \leq L_{l}=K$ be a $G_{0}$-seminormal chain of length $l$ so, for $0 \leq j<l, L_{j}=G_{0} M_{j}$ with $M_{j}$ a normal subgroup of $L_{j+1}$. Suppose first that $h+l=2$. Then $h=l=1$. In this case $N_{0}$ and $M_{0}$ are normal subgroups of $K$, so by Lemma 2.2, $(H, H \cap L)$ and $(L, H \cap L)$ are $G_{0}$-normal, and hence $(K, H \cap L)$ is $G_{0}$-seminormal of length at most $2=h+l$. Thus part (b) holds if $h+l=2$. Now we assume that $h+l>2$ and that (b) holds for smaller values of $h+l$.

Since $\left(K, L_{l-1}\right)$ is $G_{0}$-normal, it follows from Lemma 2.2 that $\left(H, H \cap L_{l-1}\right)$ is $G_{0}$-normal. Note also that $\left(H \cap L_{l-1}\right) \cap L=H \cap L$. Now $L=L_{0} \leq L_{1} \leq \cdots \leq L_{l-1}$ is a $G_{0}$-seminormal chain, and so $\left(L_{l-1}, L\right)$ is $G_{0}$-seminormal of length at most $l-1$. Also, by part (a), ( $\left.L_{l-1}, H \cap L_{l-1}\right)$ is $G_{0}$-seminormal of length at most $h$. Thus, the inductive hypothesis holds for the pairs $\left(L_{l-1}, L\right)$ and $\left(L_{l-1}, H \cap L_{l-1}\right)$, and since $\left(H \cap L_{l-1}\right) \cap L=H \cap L$, we find by induction that $\left(H \cap L_{l-1}, H \cap L\right),(L, H \cap L)$ and $\left(L_{l-1}, H \cap L\right)$ are $G_{0}$-seminormal pairs of lengths at most $l-1, h$, and $h+(l-1)$ respectively. Since $\left(H, H \cap L_{l-1}\right)$ and $\left(K, L_{l-1}\right)$ are both $G_{0}$-normal, we have that $(H, H \cap L)$ and $(K, H \cap L)$ are $G_{0}$-seminormal pairs of lengths at most $l$ and $h+l$ respectively. Thus part (b) is proved by induction.

Finally, part (c) follows from part (b) on taking $K=G$.

We show in part (b) of the next result that Seminorm is a subgroup lattice function on $\mathscr{X}_{\text {fnite }}^{\text {Seminom }}$ and its basic components are precisely the quasiprimitive groups. 
Proposition 2.4. Let $\left(G, G_{0}\right) \in \mathscr{X}_{\text {finite }}^{\text {Seminorm }}$, and let $H, L \in \operatorname{Seminorm}\left(G, G_{0}\right)$.

(a) Then $H$ and $L$ have a supremum in $\operatorname{Seminorm}\left(G, G_{0}\right)$, and if $H<L$ then Seminorm $(L, H)$ contains $\left\{K \in \operatorname{Seminorm}\left(G, G_{0}\right) \mid H \leq K \leq L\right\}$.

(b) Seminorm $\left(G, G_{0}\right)$ is a subgroup lattice containing $G$ and $G_{0}$. Moreover, all Seminorm-basic components of $\operatorname{Trans}\left(G, G_{0}\right)$ are quasiprimitive.

(c) If $\operatorname{Trans}(X, Y)$ is an arbitrary quasiprimitive group, then $(X, Y)$ lies in $\mathscr{X}_{\text {finite }}^{\text {Seminorm }}$ and $\operatorname{Seminorm}(X, Y)=\{X, Y\}$.

PROOF. Note that both $G$ and $G_{0}$ lie in $\operatorname{Seminorm}\left(G, G_{0}\right)$, since $G=G_{0} N$ with $N=G$, and $G_{0}=G_{0} N$ with $N=1$. Let

$$
\mathscr{S}=\left\{K \in \operatorname{Seminorm}\left(G, G_{0}\right) \mid\langle H, L\rangle \leq K\right\}
$$

Since $G \in \mathscr{S}$, this set is non-empty, and since $\left(G, G_{0}\right) \in \mathscr{X}_{\text {finite }}^{\text {Seminorm }}, \mathscr{S}$ is finite. It therefore follows from Proposition 2.3 (c) that $S:=\bigcap_{K \in \mathscr{S}} K$ belongs to Seminorm $\left(G, G_{0}\right)$. Since $S$ contains $\langle H, L\rangle$ it follows that $S$ is the supremum of $H$ and $L$ in $\operatorname{Seminorm}\left(G, G_{0}\right)$.

Now suppose that $H \leq L$, and let $K \in \operatorname{Seminorm}\left(G, G_{0}\right)$ such that $H \leq K \leq L$. By Proposition $2.3(\mathrm{~b}),(L, K)$ is $G_{0}$-seminormal. Let

$$
K=K_{0} \leq K_{1} \leq \cdots \leq K_{k}=L
$$

be a $G_{0}$-seminormal chain. Then, for each $i<k, K_{i}=G_{0} M_{i}$ for some normal subgroup $M_{i}$ of $K_{i+1}$. Since $H \leq K$, we have $K_{i}=G_{0} M_{i}=H M_{i}$, so the displayed chain is also $H$-seminormal. Hence $K \in \operatorname{Seminorm}(L, H)$. The proof of part (a) is now complete.

It follows from part (a) and Proposition 2.3 that $\operatorname{Seminorm}\left(G, G_{0}\right)$ is a subgroup lattice. Thus Seminorm is a subgroup lattice function on $\mathscr{X}_{\text {finite }}^{\text {Seminorm }}$.

Suppose now that $L$ covers $H$ so that $\operatorname{Trans}(L, H)$ is a Seminorm-basic component of $\operatorname{Trans}\left(G, G_{0}\right)$. Then $(L, H)$ is $G_{0}$-normal so $H=G_{0} N$, for some normal subgroup $N$ of $L$. Let $M$ be an arbitrary normal subgroup of $L$. Then $H M=G_{0}(N M)$ and $N M \triangleleft L$, so $(L, H M)$ is $G_{0}$-normal, whence $H M \in \operatorname{Seminorm}\left(G, G_{0}\right)$. Since $H \leq H M \leq L$ and $L$ covers $H$ in $\operatorname{Seminorm}\left(G, G_{0}\right)$, it follows that $H M=H$ or $H M=L$, and hence $M$ induces a trivial or transitive action on $[L: H]$, respectively. Thus $\operatorname{Trans}(L, H)$ is quasiprimitive.

Suppose finally that $\operatorname{Trans}(X, Y)$ is an arbitrary quasiprimitive permutation group, and let $H \in \operatorname{Seminorm}(X, Y)$ with $H \neq X$. Then there exists a $Y$-seminormal chain $H=H_{0}<\cdots<H_{h}=X$ with $h \geq 1$ and $H_{h-1}$ a proper subgroup of $X$. Then $H_{h-1}=Y N$, for some normal subgroup $N$ of $X$. If $N$ acts nontrivially on $[X: Y]$, then $N$ is transitive since Trans $(X, Y)$ is quasiprimitive, and hence $X=Y N=H_{h-1}$, which is a contradiction. Thus $N$ acts trivially on $[X: Y]$, and in particular is contained in the 
stabiliser $Y$. This implies that $H_{h-1}=Y=H$. Hence Seminorm $(X, Y)=\{X, Y\}$, and in particular $(X, Y) \in \mathscr{X}_{\text {finite }}^{\text {Seminorm }}$.

The proof of Theorem 1.12 follows from Proposition 2.4.

\section{The subnormal lattice function.}

Since $G$ is a normal subgroup of itself it follows that $G \in \operatorname{Subnorm}\left(G, G_{0}\right)$. Suppose that $H \in \operatorname{Subnorm}\left(G, G_{0}\right)$ and $H \neq G$. Then, by definition, $H=G_{0} N$ for some proper, $G_{0}$-invariant, composition subgroup $N$ of $G$, and so we have a composition series from $N$ to $G$, say

$$
N=N_{0} \triangleleft N_{1} \triangleleft \cdots \triangleleft N_{h}=G
$$

for some $h \geq 1$, where each $N_{i+1} / N_{i}$ is simple. Note that, although $N_{0}=N$ and $N_{h}=G$ are $G_{0}$-invariant, the other $N_{i}$ are not in general $G_{0}$-invariant. Our first task is to construct a subnormal series from $N$ to $G$ in which all the subgroups are $G_{0}$-invariant composition subgroups of $G$.

LEMMA 3.1. Let $\left(G, G_{0}\right) \in \mathscr{X}_{\text {all }}$, let $H=G_{0} N \in \operatorname{Subnorm}\left(G, G_{0}\right)$, where $H \neq G$. Then there is a $G_{0}$-seminormal chain $H=G_{0} M_{l} \leq G_{0} M_{l-1} \leq \cdots \leq G_{0} M_{0}=G$ such that $M_{0}=G, l \leq h, N \leq M_{l}$ and, for each $i>0, M_{i}$ is a normal subgroup of $M_{i-1}$ and is a $G_{0}$-invariant composition subgroup of $G$. In particular, $H \in \operatorname{Seminorm}\left(G, G_{0}\right)$.

Proof. Let the $N_{i}$ be as in (1). We shall construct inductively $G_{0}$-invariant composition subgroups $M_{0}, M_{1}, \ldots, M_{h}$ of $G$ such that $M_{h} \triangleleft M_{h-1} \triangleleft \cdots \triangleleft M_{0}=G$ (with possible equality for some inclusions), and such that, for each $i, N \leq M_{i} \leq N_{h-i}$.

The group $M_{0}:=G$ is a $G_{0}$-invariant composition subgroup containing $N$. Also $M_{1}:=N_{h-1}$ is a normal subgroup of $M_{0}=G$ and hence is a $G_{0}$-invariant composition subgroup of $G$ that contains $N$. Assume now that $1<i \leq h$ and that we have constructed $G_{0}$-invariant composition subgroups $M_{0}, M_{1}, \ldots, M_{i-1}$ such that $M_{i-1} \triangleleft$ $M_{i-2} \triangleleft \cdots \triangleleft M_{0}=G$, and $N \leq M_{j} \leq N_{h-j}$ for each $j \leq i-1$. We shall construct a $G_{0}$-invariant composition subgroup $M_{i}$ of $G$ that is a normal subgroup of $M_{i-1}$ and satisfies $N \leq M_{i} \leq N_{h-i}$. If $M_{i-1}=N$ then the subgroup $M_{i}=N$ has these properties. So assume that $N$ is a proper subgroup of $X:=M_{i-1}$. Then the distinct subgroups in the series

$$
N=N \cap X \leq N_{1} \cap X \leq \cdots \leq N_{h} \cap X=X
$$

form a composition series from $N$ to $X$. Let $Y=N_{j} \cap X$ be the largest proper subgroup of $X$ in this series. Then $Y$ is normal in $X$ and $X / Y$ is simple. Define 
$M_{i}=\bigcap_{g \in G_{0}} Y^{g}$. Since $X, N$ are $G_{0}$-invariant, each of the groups $Y^{g}$ (for $g \in G_{0}$ ) is normal in $X$ and contains $N$, and hence $M_{i}$ is a normal $G_{0}$-invariant subgroup of $X$ containing $N$. Also, since $X \leq N_{h-i+1}$, it follows from the definition of $Y$ that $Y=N_{j} \cap X \leq N_{h-i}$, and hence that $M_{i} \leq N_{h-i}$. It remains to show that $M_{i}$ is a composition subgroup of $G$. Since $M_{i}$ is a normal subgroup of $X$, each $N_{k} \cap X$ normalises $M_{i}$ and so each $M_{i}\left(N_{k} \cap X\right)$ is a subgroup, and if $k<h$ then $M_{i}\left(N_{k} \cap X\right)$ is normal in $M_{i}\left(N_{k+1} \cap X\right)$. Thus the distinct subgroups in the series

$$
M_{i}=M_{i}(N \cap X) \leq M_{i}\left(N_{1} \cap X\right) \leq \cdots \leq M_{i}\left(N_{h} \cap X\right)=X .
$$

form a composition series from $M_{i}$ to $X$, and since $X$ is a composition subgroup of $G$, it follows that $M_{i}$ is also a composition subgroup of $G$. Continuing in this way we construct all of the subgroups $M_{0}, \ldots, M_{h}$.

Then the subgroup chain $H=G_{0} M_{h} \leq G_{0} M_{h-1} \leq \cdots \leq G_{0} M_{0}=G$ is a $G_{0^{-}}$ seminormal chain with the required properties, and we can take $l$ to be the least $i$ such that $H=G_{0} M_{i}$. In particular $H \in \operatorname{Seminorm}\left(G, G_{0}\right)$.

Now we prove that $\operatorname{Subnorm}\left(G, G_{0}\right)$ is a subgroup lattice. Recall (see Example 1.14) that $\operatorname{Subnorm}\left(G, G_{0}\right)$ is not always a sublattice of $\operatorname{Sub}\left(G, G_{0}\right)$.

Proposition 3.2. Let $\left(G, G_{0}\right) \in \mathscr{X}_{\text {all }}$, and let $H, L \in \operatorname{Subnorm}\left(G, G_{0}\right)$.

(a) Then Subnorm $\left(G, G_{0}\right) \subseteq \operatorname{Seminorm}\left(G, G_{0}\right)$, and $\langle H, L\rangle$ is the supremum of $H$ and $L$ in $\operatorname{Subnorm}\left(G, G_{0}\right)$. Moreover, $\operatorname{Subnorm}\left(G, G_{0}\right)$ always contains $G$; it contains $G_{0}$ if and only if $G_{0}$ contains a $G_{0}$-invariant composition subgroup of $G$.

(b) If $\left(G, G_{0}\right) \in \mathscr{X}_{\text {finite }}^{\text {Subm, }}$, then Subnorm $\left(G, G_{0}\right)$ is a subgroup lattice.

(c) Subnorm is a subgroup lattice function on $\mathscr{X}_{\text {finite }}^{\text {Sunorm }}$, but it is not strong.

ProOF. By Lemma 3.1, Subnorm $\left(G, G_{0}\right) \subseteq \operatorname{Seminorm}\left(G, G_{0}\right)$. Now $H=G_{0} N$ and $L=G_{0} M$, where $N, M$ are $G_{0}$-invariant composition subgroups of $G$. By Wielandt's Theorem [11, Theorem 1.1.5], $X:=\langle N, M\rangle$ is a composition subgroup of $G$, and clearly $X$ is $G_{0}$-invariant. Moreover, $\langle H, L\rangle=\left\langle G_{0}, N, M\right\rangle=G_{0} X$, and hence $\langle H, L\rangle \in \operatorname{Subnorm}\left(G, G_{0}\right)$. As noted above $\operatorname{Subnorm}\left(G, G_{0}\right)$ contains $G$. By definition, Subnorm $\left(G, G_{0}\right)$ contains $G_{0}$ if and only if $G_{0}$ is of the form $G_{0} N$ for some $G_{0}$-invariant composition subgroup $N$ of $G$, or equivalently, if and only if $G_{0}$ contains such a subgroup $N$. Thus (a) is proved.

Suppose now that $\left(G, G_{0}\right) \in \mathscr{X}_{\text {finite }}^{\text {Subrm }}$. We will show that $H, L$ have an infimum in Subnorm $\left(G, G_{0}\right)$. Let $\mathscr{S}$ be the set of all subgroups in $\operatorname{Subnorm}\left(G, G_{0}\right)$ that are contained in $H \cap L$. Since $N \cap M$ is a $G_{0}$-invariant composition subgroup of $G$ it follows that $G_{0}(N \cap M)$ is a subgroup of $H \cap L$ that lies in $\operatorname{Subnorm}\left(G, G_{0}\right)$, so $\mathscr{S}$ is nonempty. Since $\left(G, G_{0}\right) \in \mathscr{Q}_{\text {finite }}^{\text {-Subnorm }}$, Subnorm $\left(G, G_{0}\right)$ is finite and hence $\mathscr{S}$ is finite, say $\mathscr{S}=\left\{G_{0} S_{1}, \ldots, G_{0} S_{k}\right\}$ where each of the $S_{i}$ is a $G_{0}$-invariant composition subgroup of $G$. Let $Y:=\left\langle S_{1}, \ldots, S_{k}\right\rangle$. By [11, Theorem 1.1.5] again, $Y$ is a composition 
subgroup of $G$, and since each $S_{i}$ is $G_{0}$-invariant and contained in $H \cap L$, it follows that $Y$ is $G_{0}$-invariant and contained in $H \cap L$. This means that $G_{0} Y \in \operatorname{Subnorm}\left(G, G_{0}\right)$ and $G_{0} Y \leq H \cap L$. By definition therefore $G_{0} Y \in \mathscr{S}$, and $G_{0} Y$ contains all other subgroups in $\mathscr{S}$. Thus $G_{0} Y$ is the infimum of $H$ and $L$ in Subnorm $\left(G, G_{0}\right)$. It now follows from this fact, and from part (a) that $\operatorname{Subnorm}\left(G, G_{0}\right)$ is a subgroup lattice. Thus part (b) is proved.

By part (b), Subnorm is a subgroup lattice function on $\mathscr{X}_{\text {finite }}^{\text {Suborm }}$, and by Example 1.14 , it is not strong.

Now we look at the roles of quasiprimitive and innately transitive permutation groups in describing Subnorm-components, proving in particular that every finite Subnorm-basic component is innately transitive.

Proposition 3.3. Let $\left(G, G_{0}\right) \in \mathscr{X}_{\text {all }}$ be such that there is some $G_{0}$-invariant composition subgroup of $G$ contained in $G_{0}$.

(a) Then $\left\{G, G_{0}\right\} \subseteq$ Subnorm $\left(G, G_{0}\right)$, and equality holds if and only if the permutation group $\operatorname{Trans}\left(G, G_{0}\right)$ is quasiprimitive.

(b) Each finite Subnorm-basic component of $\operatorname{Subnorm}\left(G, G_{0}\right)$ is innately transitive.

ProOF. By Proposition 3.2 (a), $\left\{G, G_{0}\right\} \subseteq \operatorname{Subnorm}\left(G, G_{0}\right) \subseteq \operatorname{Seminorm}\left(G, G_{0}\right)$. Suppose that $\operatorname{Trans}\left(G, G_{0}\right)$ is quasiprimitive. Then by Proposition 2.4 (c), we have $\operatorname{Seminorm}\left(G, G_{0}\right)=\left\{G, G_{0}\right\}$, and hence also $\operatorname{Subnorm}\left(G, G_{0}\right)=\left\{G, G_{0}\right\}$. Conversely, suppose that $\operatorname{Subnorm}\left(G, G_{0}\right)=\left\{G, G_{0}\right\}$. We need to prove that the group $\operatorname{Trans}\left(G, G_{0}\right)$ is quasiprimitive. First we show that the kernel $K$ of the action of $G$ on [G: $\left.G_{0}\right]$ is a composition subgroup of $G$. By Lemma 3.1, there is a $G_{0}$-seminormal chain

$$
G_{0}=G_{0} M_{l}<G_{0} M_{l-1}<\cdots<G_{0} M_{0}=G
$$

such that $l \geq 1, M_{0}=G$, and, for each $i=1, \ldots, l, M_{i}$ is a normal subgroup of $M_{i-1}$ and is a $G_{0}$-invariant composition subgroup of $G$. By definition of Subnorm, each of the $G_{0} M_{i}$ lies in $\operatorname{Subnorm}\left(G, G_{0}\right)=\left\{G, G_{0}\right\}$, and hence $l=1$ so that $G_{0}=G_{0} M_{1}$, with $M_{1}$ a composition subgroup of $G$ that is normal in $G$. Since $M_{1} \leq G_{0}$ and $M_{1}$ is normal in $G$, it follows that $M_{1} \leq K$. Then since $M_{1}$ is a composition subgroup and $K$ is a normal subgroup of $G$ containing $M_{1}$, it follows that $K$ is a composition subgroup of $G$. Now to each normal subgroup $Y$ of $\operatorname{Trans}\left(G, G_{0}\right) \cong G / K$ there corresponds a unique normal subgroup $X$ of $G$ containing $K$ such that $Y \cong X / K$. Since $K$ is a composition subgroup of $G$, it follows that $X$ is also a composition subgroup of $G$. Since $X$ is normal in $G$, it is $G_{0}$-invariant, and hence $G_{0} X \in \operatorname{Subnorm}\left(G, G_{0}\right)=$ $\left\{G, G_{0}\right\}$. If $G_{0} X=G_{0}$ then $X \subseteq G_{0}$, and since the kernel $K$ is the largest normal subgroup of $G$ contained in $G_{0}$, it follows that in this case $X=K$, and so $Y=1$. On 
the other hand if $G_{0} X=G$, then $X$ acts transitively on [ $G: G_{0}$ ], and hence in this case $Y$ is transitive. Thus $\operatorname{Trans}\left(G, G_{0}\right)$ is quasiprimitive, and so part (a) is proved.

Now suppose that $K, H \in \operatorname{Subnorm}\left(G, G_{0}\right)$ and that $K$ covers $H$, so that $\operatorname{Trans}(K, H)$ is a Subnorm-basic component of $\operatorname{Trans}\left(G, G_{0}\right)$. Suppose further that $\operatorname{Trans}(K, H)$ is finite. Let $Y=\operatorname{Core}_{K}(H)$, so that $\operatorname{Trans}(K, H) \cong K / Y$. We have $H=G_{0} N$ anc $K=G_{0} M$, where $M, N$ are $G_{0}$-invariant composition subgroups of $G$. Thus $M$ is a normal subgroup of $K$, and so $M Y / Y$ is a normal subgroup of the finite group $K / Y$. Hence $M /(M \cap Y) \cong M Y / Y$ is finite, and $M /(M \cap Y)$ is normal in $K /(M \cap Y)$. Thus there exists a normal subgroup $L$ of $K$ such that $M \cap Y<L \leq M$, and $L /(M \cap Y)$ is a minimal normal subgroup of $K /(M \cap Y)$. The corresponding subgroup $L Y / Y$ of $K / Y$ is therefore a minimal normal subgroup of $K / Y$ contained in $M Y / Y$. Now $L$ is $H$-invariant since $L$ is normal in $K$, and so we have $H \leq H L \leq K$, and we note that $H L=G_{0}(N L)$, and $N$ normalises $L$. The subgroup $L$ is a composition subgroup of $G$ since $M$ is a composition subgroup and $L$ is a normal subgroup of $M$ of finite index. Then, since both $N$ and $L$ are $G_{0}$-invariant composition subgroups, it follows that $\langle N, L\rangle=N L$ is a $G_{0}$-invariant composition subgroup (using Wielandt's Theorem again). Hence $H L=G_{0}(N L) \in \operatorname{Subnorm}\left(G, G_{0}\right)$, and since $K$ covers $H$ it follows that $H L$ is $H$ or $K$. If $H L=H$ then $L \leq H$, and since $L$ is normal in $K$ this implies that $L \leq$ Core $_{K}(H)=Y$, which is a contradiction. Hence $H L=K$, and this implies that $L Y / Y$ is transitive. Thus $\operatorname{Trans}(K, H)$ has a transitive minimal normal subgroup and so is innately transitive.

There is one further ingredient to put in place to enable us to complete the proof of Theorem 1.13, namely we must demonstrate that each finite innately transitive group occurs as a Subnorm-basic component. To do this we use a construction similar to the construction given in [2, Construction 6.6] for innately transitive groups. For a group $K$ and subgroup $K_{0}$, $\operatorname{Inn}(K)$ denotes the group of inner automorphisms of $K$, and $\operatorname{Inn}_{K_{0}}(K)$ denotes the subgroup of $\operatorname{Inn}(K)$ induced by elements of $K_{0}$. We use $A: B$ to denote a semidirect product of $A$ by $B$.

\section{CONSTRUCTION 3.4. Let}

(a) $K=T^{k}$, where $k \geq 1$, and $T$ is a finite simple group (possibly abelian),

(b) $J \triangleleft K_{0} \leq K$ such that Core ${ }_{K}(J)=1$, and if $K$ is abelian, then $K_{0}=K$,

(c) $L \leq \operatorname{Aut}(K)$ such that the only $L$-invariant normal subgroups of $K$ are 1 and $K$, and such that $L \cap \operatorname{Inn}(K)=\operatorname{Inn}_{K_{0}}(K)$, and $J$ is $L$-invariant.

Define $G$ to be the semidirect product $(K \times K): L$, where $L$ acts naturally on each of the two direct factors of $K \times K$, let $G_{0}=Y: L$, where

$$
Y=\left\{(x, y) \mid x, y \in K_{0}, J x=J y\right\},
$$

and let $A=G_{0}(1 \times K)=\left(K_{0} \times K\right): L$. 
Proposition 3.5. Let $G, G_{0}, A$ be as in Construction 3.4, and let $\varphi$ be the nat-

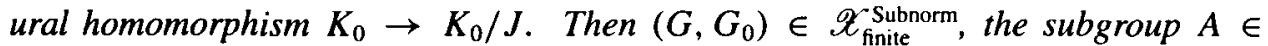
Subnorm $\left(G, G_{0}\right)$, and $\operatorname{Trans}\left(A, G_{0}\right)$ is a Subnorm-basic component of $\operatorname{Trans}\left(G, G_{0}\right)$. Moreover, $\operatorname{Trans}\left(A, G_{0}\right)$ is permutationally isomorphic to the innately transitive group constructed from the triple $(K, \varphi, L)$ in [2, Construction 6.6]; and every finite innately transitive group arises in this way.

Proof. Since $G$ is finite and $G_{0} \neq G$, we have $\left(G, G_{0}\right) \in \mathscr{X}_{\text {finite }}^{\text {Subnorm }}$, and since $1 \times K$ is normal in $G$, it follows that $A \in \operatorname{Subnorm}\left(G, G_{0}\right)$. Next we prove that $A$ covers $G_{0}$ in $\operatorname{Subnorm}\left(G, G_{0}\right)$. Suppose that $H \in \operatorname{Subnorm}\left(G, G_{0}\right)$ and $G_{0} \leq H<A$.

Then $H=G_{0} N$ for some $G_{0}$-invariant composition subgroup $N$ of $G$. Let $M=(1 \times K) N$. Then $M$ is a composition subgroup of $G$, by Wielandt's Theorem, and $A=G_{0} M$ since $N \leq A$. It follows from Lemma 3.1, and its proof, that there is a subnormal chain

$$
M_{l}<M_{l-1} \leq \cdots \leq M_{0}=M
$$

where $l \geq 1, H=G_{0} N=G_{0} M_{l}, N \leq M_{0}$, each of the $M_{i}$ is $G_{0}$-invariant, and if $i>0$ then $M_{i}$ is a proper normal subgroup of $M_{i-1}$. We may suppose that this chain is maximal with respect to these properties. Then $U:=M_{1}$ is a proper normal subgroup of $M$ containing $N$, and there is no $G_{0}$-invariant normal subgroup $V$ of $M$ such that $U<V<M$. Since $U \neq M$ it follows that $U$ does not contain $1 \times K$. Since $U$ is normalised by $M$, we have that $U \cap(1 \times K)$ is a normal subgroup of $1 \times K$; and since $U$ is $G_{0}$-invariant it follows that $U \cap(1 \times K)$ is $L$-invariant. Thus, using condition (c), we deduce that $U \cap(1 \times K)=1$. In particular, $U$ centralises $1 \times K$, and $(1 \times K) U$ is a $G_{0}$-invariant normal subgroup of $M$ properly containing $U$. Thus $U \leq C_{G}(1 \times K)$, and using condition (c) again, we have $M \cong U \times(1 \times K)$.

Since $M=(1 \times K) N$ and $N \leq U \cong M /(1 \times K)$, it follows that $N=U$. Hence $N \leq C_{G}(1 \times K)$. We claim that $C_{G}(1 \times K)=\left\{(x, y) \iota_{y}^{-1} \mid x \in K, y \in K_{0}\right\}$ (where $\iota_{y}$ is the inner automorphism of $K$ induced by $\left.y\right)$. Let $(x, y) \sigma \in C_{G}(1 \times K)$, where $x, y \in K$ and $\sigma \in L$. For $(1, u) \in 1 \times K,(1, u)^{(x, y) \sigma}=\left(1, u^{y \sigma}\right)$, and it follows that $\sigma=\iota_{y}^{-1}$. By condition (c), $L \cap \operatorname{Inn}(K)=\operatorname{Inn}_{K_{0}}(K)$, and hence $y \in K_{0}$. Conversely each element $(x, y) \iota_{y}^{-1}$, with $x \in K, y \in K_{0}$, centralises $1 \times K$. Thus the claim is proved, and we have that

$$
\begin{aligned}
N \leq C_{G}(1 \times K) \cap A & =C_{G}(1 \times K) \cap\left(\left(K_{0} \times K\right): L\right) \\
& =\left\{(x, y) \iota_{y}^{-1} \mid x, y \in K_{0}\right\} .
\end{aligned}
$$

Now $K \times 1$ is normal in $G$ and hence $M^{\prime}:=(K \times 1) N$ is also a $G_{0}$-invariant composition subgroup of $G$ containing $N$ (by Wielandt's Theorem). Thus there is a $G_{0}$-invariant subnormal chain from $N$ to $M^{\prime}$; let $U^{\prime}$ be the largest proper subgroup of $M^{\prime}$ in this chain. An analogous argument to the one above, with $M^{\prime}, U^{\prime}, K \times 1$ 
in place of $M, U, 1 \times K$, shows that $U^{\prime}$ centralises $K \times 1$, that $U^{\prime}=N$, and that $C_{G}(K \times 1)=\left\{(x, y) \iota_{x}^{-1} \mid x \in K_{0}, y \in K\right\}$. Combining this information with (2), we obtain that $N \leq\left\{(x, x) \iota_{x}^{-1} \mid x \in K_{0}\right\}$. Thus $N \leq G_{0}$, and hence $G_{0} N=G_{0}$, so $A$ covers $G_{0}$ in Subnorm $\left(G, G_{0}\right)$.

It follows that $\operatorname{Trans}\left(A, G_{0}\right)$ is a Subnorm-basic component of $\operatorname{Trans}\left(G, G_{0}\right)$. Now $\operatorname{Trans}\left(A, G_{0}\right)$ is the permutation group induced by the right multiplication action of $A$ on $\left[A: G_{0}\right.$ ]. Moreover, $G_{0}$ contains the normal subgroup $J \times 1$ of $A$. Let $\varphi$ be the natural homomorphism $\varphi: K_{0} \rightarrow K_{0} / J$, so that $K_{0}, J$ are the domain and kernel of $\varphi$, respectively. The conditions (a)-(c) on $K, K_{0}, J, L$ in Construction 3.4 give precisely the conditions for $(K, \varphi, L)$ to be an innate triple, as defined in [2, Definition 6.1]. Moreover, $X:=(\operatorname{Im} \varphi \times K): L \cong A /(J \times 1)$ is the group constructed in [2, Construction 6.6] from the triple $(K, \varphi, L)$, and the subgroup of $X$ corresponding to $G_{0} /(J \times 1)$ is $X_{0}:=\left\{(\varphi(u), u) \mid u \in K_{0}\right\}: L$. Thus $\operatorname{Trans}\left(A, G_{0}\right)$ is permutationally isomorphic to the group induced by $X$ on $\left[X: X_{0}\right]$, and by [2, Proposition 6.7], this is the innately transitive group constructed from $(K, \varphi, L)$ in [2, Construction 6.6].

By [2, Theorem 1.1], up to permutational isomorphism, every finite innately transitive group arises from [2, Construction 6.6] applied to some triple $(K, \varphi, L)$ satisfying the conditions in Construction 3.4 (with $K_{0}, J$ the domain and kernel of $\varphi$ ). This is because every epimorphism with domain $K_{0}$ and kernel $J$ can be replaced in the construction in [2, Construction 6.6] by the natural map $K_{0} \rightarrow K_{0} / J$. Thus, by [2, Theorem 1.1], every finite innately transitive group arises as $\operatorname{Trans}\left(A, G_{0}\right)$ for some $G, G_{0}$ in Construction 3.4 .

Theorem 1.13 follows from Propositions 3.2,3.3, and 3.5.

\section{Acknowledgement}

I wish to thank an anonymous referee for pointing out three inaccuracies in an earlier version of this paper (now corrected).

\section{References}

[1] R. A. Bailey, C. E. Praeger, C. A. Rowley and T. P. Speed, 'Generalized wreath products of permutation groups', Proc. London Math. Soc. (3) 47 (1983), 69-82.

[2] J. Bamberg and C. E. Praeger, 'Finite transitive permutation groups with a transitive minimal normal subgroup', Proc. London Math. Soc. 89 (2004), 71-103.

[3] M. Bhattacharjee, D. Macpherson, R. G. Möller and P. M. Neumann, Notes on infinite permutation groups (Hindustan book agency. New Delhi, 1997).

[4] F. Buekenhout, A. Delandtsheer, J. Doyen, P. B. Kleidman, M. W. Liebeck and J. Saxl, 'Linear spaces with flag-transitive automorphism groups', Geometriae Dedicata 36 (1990), 89-94. 
[5] A. R. Camina and C. E. Praeger, 'Line-transitive, point-quasiprimitive automorphism groups of finite linear spaces are affine or almost simple', Aequationes Math. 61 (2001), 221-232.

[6] J. D. Dixon and B. Mortimer, Permutation groups (Springer, New York, 1996).

[7] J. C. Lennox and S. E. Stonehewer, Subnormal subgroups of groups (Clarendon Press, Oxford, 1987).

[8] C. E. Praeger, 'An O'Nan-Scott theorem for finite quasiprimitive permutation groups and an application to 2-arc transitive graphs', J. London Math. Soc. (2) 47 (1993), 227-239.

[9] — 'Finite quasiprimitive graphs', in: Surveys in Combinatorics, 1997 (London) (ed. R. A. Bailey), London Math. Soc. Lecture Note Ser. 241 (Cambridge Univ. Press, Cambridge, 1997) pp. 65-85.

[10] C. E. Praeger, J. Saxl and K. Yokoyama, 'Distance transitive graphs and finite simple groups', Proc. London Math. Soc. (3) 55 (1987), 1-21.

[11] R. Schmidt, Subgroup lattices of groups (de Gruyter, Berlin, 1994).

School of Mathematics and Statistics

The University of Western Australia

35 Stirling Highway

Crawley WA 6009

Australia

e-mail: praeger@maths.uwa.edu.au 
